University of Nebraska - Lincoln

DigitalCommons@University of Nebraska - Lincoln

Faculty Publications, Department of Physics and Astronomy

Research Papers in Physics and Astronomy

2005

\title{
Experimental Studies of Light Propagation and Storage in Warm Atomic Gases
}

H. Gao

University of Nebraska - Lincoln

Mark A. Rosenberry

Siena College, mrosenberry@siena.edu

J. Wang

University of Nebraska - Lincoln

Herman Batelaan

University of Nebraska - Lincoln, hbatelaan@unl.edu

Follow this and additional works at: https://digitalcommons.unl.edu/physicsfacpub

Part of the Physics Commons

Gao, H.; Rosenberry, Mark A.; Wang, J.; and Batelaan, Herman, "Experimental Studies of Light Propagation and Storage in Warm Atomic Gases" (2005). Faculty Publications, Department of Physics and Astronomy. 102.

https://digitalcommons.unl.edu/physicsfacpub/102

This Article is brought to you for free and open access by the Research Papers in Physics and Astronomy at DigitalCommons@University of Nebraska - Lincoln. It has been accepted for inclusion in Faculty Publications, Department of Physics and Astronomy by an authorized administrator of DigitalCommons@University of Nebraska Lincoln. 


\title{
Experimental studies of light propagation and storage in warm atomic gases
}

\author{
H Gao $^{1}$, M Rosenberry ${ }^{2}, \mathrm{~J} \mathrm{Wang}^{1}$ and H Batelaan ${ }^{1}$ \\ ${ }^{1}$ Department of Physics and Astronomy, University of Nebraska-Lincoln, 116 Brace Lab, \\ Lincoln, NE 68588-0111, USA \\ 2 Department of Physics, Sienna College, 515 Loudon Road, Loudonville, NY 12211-1462, USA
}

Received 2 January 2005

Published 6 June 2005

Online at stacks.iop.org/JPhysB/38/1857

\begin{abstract}
We report on an experimental study of slow pulse propagation and polarization storage of light in an atomic vapour for pulse durations ranging from several $\mu \mathrm{s}$ to $100 \mu \mathrm{s}$, for non-zero magnetic fields, for temperatures ranging from 65 to $90{ }^{\circ} \mathrm{C}$ and for two different rubidium isotopes. The results confirm the presence of the electromagnetically induced transparency window as predicted by Lukin (1997 Phys. Rev. Lett. 79 2959). We show that time-dependent optical rotation can mask the storage of light signal, and demonstrate storage of light for the ${ }^{85} \mathrm{Rb}$ isotope.
\end{abstract}

\section{Introduction}

The resonant interaction of light with three-level $\Lambda$-type atoms has attracted considerable attention in recent years. Due to the dramatic variation of the index of refraction of the atomic vapour close to resonance, many interesting effects occur [1-9]. Important examples are electromagnetically induced transparency (EIT) [1] and ultra slow propagation of light $[2,6]$. The EIT effect and the low group velocity of a pulse of light have both been studied thoroughly. With the combination of these two techniques, certain properties of the light pulse can be stored with modest absorption in the atomic ensemble, as has been demonstrated recently [10-19]. Other storage media [20,21] and schemes not relying on a $\Lambda$-type atoms $[22,23]$ are also being explored.

In the field of quantum information, the light storage technique may provide a feasible way to realize a quantum memory for photons [15], high-efficiency photon counting [24] and probe ultra cold gasses [25, 26]. In the field of classical optics, the light slowing and storage techniques may lead to new types of delay lines [21], and ultra-sensitive Sagnac interferometers [27]. There are a large number of experimental parameters that affect storage of light. In a previous study [18] we explored the dependence on light polarization and showed that all polarizations can be stored with high fidelity. The purpose of this paper is to study the experimental parameters that are readily accessible and provide useful information for researchers starting to build a light slowing/storage apparatus. The parameters studied in this paper are signal pulse duration, magnetic field strength, temperature and isotope. 
Recent theoretical studies show $[10,15]$ that the spectral width of the pulse should be contained within the EIT transparency window to avoid loss and dissipation. This is known as the adiabatic condition. If the pulse is too short then the response of the atomic system should not setup EIT within the signal pulse duration. By reducing the light pulse duration, the frequency width of the pulse can be made increasingly larger than the EIT transparency window. We study how much dissipation occurs, and how the light storage is affected. In section 4.1 we report on the observation of storage of light for pulse durations of several $\mu$ s to $100 \mu \mathrm{s}$, including pulse durations that do not satisfy the adiabatic condition.

The external magnetic field can directly change the atomic EIT properties. On the other hand, nonlinear magneto-optic rotation (NMOR) can also occur in the resonant interaction of light with atoms in a $\Lambda$-type atomic structure. NMOR is an optical rotation effect and can be used in high-precision laser spectroscopy and magnetometry [5-9]. Typically, optical rotation is studied that is caused by an atomic system in the steady state. However, in storage experiments the laser is switched and leads the time-dependent optical rotation effects. It is important in this study to distinguish the time-dependent optical rotation effects from the light storage itself. This means that observing a time-dependent signal with the two peaks that look like the typical pre-storage and post-storage pulse, does not necessarily mean that storage has been observed. The act of switching on the control laser can produce a peak that looks like a post-storage signal, even in the absence of a signal pulse. For linear polarization it is not easy to distinguish the light storage from the optical rotation when an ambient magnetic field is present. In section 4.2 we report on our finding that optical rotation occurs at the time when the control light switches on again, which results in a nontrivial storage signal. To avoid this, good magnetic shielding (within $2 \mathrm{mG}$ ) is necessary.

The EIT depends on the atomic density. At low Rb cell temperatures the smaller atomic density makes the EIT signal too weak to be detected. At high $\mathrm{Rb}$ cell temperatures the absorption of the laser pulse is too strong to allow a signal to be observed. In section 4.3 we report on our finding that the working temperature ranges from $65-90{ }^{\circ} \mathrm{C}$. We also show that the atomic density governs the width of the EIT transparency window.

Finally, by comparing light storage for the ${ }^{87} \mathrm{Rb}$ isotope to storage in the ${ }^{85} \mathrm{Rb}$ isotope, one might expect a stronger signal, since the ${ }^{85} \mathrm{Rb}$ isotope is more abundant than the ${ }^{87} \mathrm{Rb}$ isotope. In section 4.4 we demonstrate storage in ${ }^{85} \mathrm{Rb}$, but find that it is typically weaker than storage for the ${ }^{87} \mathrm{Rb}$ isotope.

We have organized the numbering of our figures in groups. Pulse width effects (section 4.1) are shown in figure 2, magnetic field effects (section 4.2) are shown in figure 3, temperaturedependent effects (section 4.3) are shown in figure 4, and the isotope dependent effect is given in figure 5. All figures numbers 2, 3, 4(a) refer to light slowing, while figures 2, 3, 4(b) refer to light storage.

\section{Experimental setup}

The schematical experimental setup is shown in figure 1, and is similar to other recent light storage experiments [12, 16-18]. Throughout this study we used linearly polarized light, unless otherwise indicated. An external cavity diode laser with a bandwidth of less than $300 \mathrm{kHz}$ is used as the linearly polarized light source. An acoustic-optical modulator (AOM) and iris allow for rapid switching of this source. The linear polarization can be slightly rotated by a fast Pockel's cell ( $\sim 100 \mathrm{~ns})$ to create a weak pulse. As usual this will be called the signal field. The polarization of the signal field is perpendicular to that of the remaining light, which serves as the control field. Linearly polarized light couples the ground states, to the excited states. Our Rb cell is $4 \mathrm{~cm}$ long, $2 \mathrm{~cm}$ in diameter and contains about 5 Torr of helium buffer 


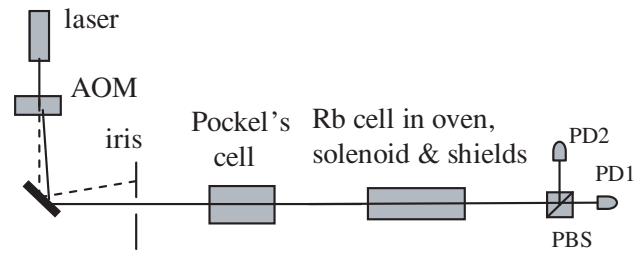

Figure 1. Schematic of the experimental setup. PD indicates a photodiode. Other components are defined in the text.

gas. A solenoid is placed around the Rb cell to precisely control the static magnetic field along the propagation direction of the laser beam. The solenoid is enclosed within double layered magnetic shielding to insure long lifetimes of the atomic Zeeman coherence. The atomic density can be varied by an Aerobiax heater cable, which is wrapped around the magnetic shielding. A polarizing beam splitter and two photodiodes, indicated with PBS and PD1/PD2 in figure 1, comprise the detection system. The PBS separates the control and signal fields after the light has passed the Rb cell, to be detected at the two photodiodes. To create the EIT effect, the laser frequency is adjusted to the $\mathrm{D} 1$ transition for ${ }^{87} \mathrm{Rb}(\lambda=794.987 \mathrm{~nm})$, i.e., $5 \mathrm{~S}_{1 / 2}, F=2 \rightarrow 5 \mathrm{P}_{1 / 2}, F=1$, which is checked by observing the fluorescence and absorption spectrum. Alternatively, the laser is adjusted to the D1 transition for ${ }^{85} \mathrm{Rb}(\lambda=794.984 \mathrm{~nm})$, i.e., $5 \mathrm{~S}_{1 / 2}, F=3 \rightarrow 5 \mathrm{P}_{1 / 2}, F=2$. The laser beam diameter is about $\sim 5 \mathrm{~mm}$ with an output power of $\sim 8 \mathrm{~mW}(2.5 \mathrm{~mW}$ incident on the cell). The level scheme of the transitions involved is complex. When using circularly polarized light, the atoms are optically pumped into the highest magnetic substate. This justifies the use of simplified three state $\Lambda$-type level structure. However, for the linearly polarized case we do not have this luxury. All magnetic substates of the $\mathrm{Rb}$ ground state are populated.

\section{Theoretical considerations}

First we give the general framework in which our experimental results can be understood. It will turn out that the simplified $\Lambda$-type structure is still sufficient to understand some qualitative trends in the experimental data, even though the light is linearly polarized. We may think of the linearly polarized light as two orthogonal circularly polarized components. This motivates the choice of using the usual $\Lambda$-type structure to analyse this system. A more complete analysis including more states is beyond the scope of this paper. Complete level schemes including their coupling for circularly and linearly polarized light are beautifully described in [28]. More detailed theoretical studies on light propagation and storage in three-level systems can be found in $[15,29,30]$. Typically, the susceptibility, $\chi$, for a resonant laser field propagating through an ideal, homogeneous EIT medium can be expressed as [3, 15],

$$
\chi=\kappa \gamma \frac{\delta}{\Omega^{2}-\delta^{2}-\mathrm{i} \gamma \delta},
$$

where $\kappa=3 N \lambda^{3} / 8 \pi^{2}$ is a constant, $\lambda$ is the resonant laser wavelength, $\gamma$ is the radiative decay rate of the excited state to the ground state, $\Omega$ is the Rabi frequency and $\delta=\omega-\omega_{0}$ is the laser detuning. The dispersion relation for light propagating in such a medium, $(k \mathrm{c} / \omega)^{2}=1+\chi$, can be found by substituting plane waves into the wave equation. This can be reduced to

$$
k \mathrm{c} / \omega=1+\chi / 2,
$$

for small values of the susceptibility near resonance. Here, $\omega$ and $k$ are the laser frequency and wave number, and $c$ is the speed of light in vacuum. Generally, the susceptibility is divided 

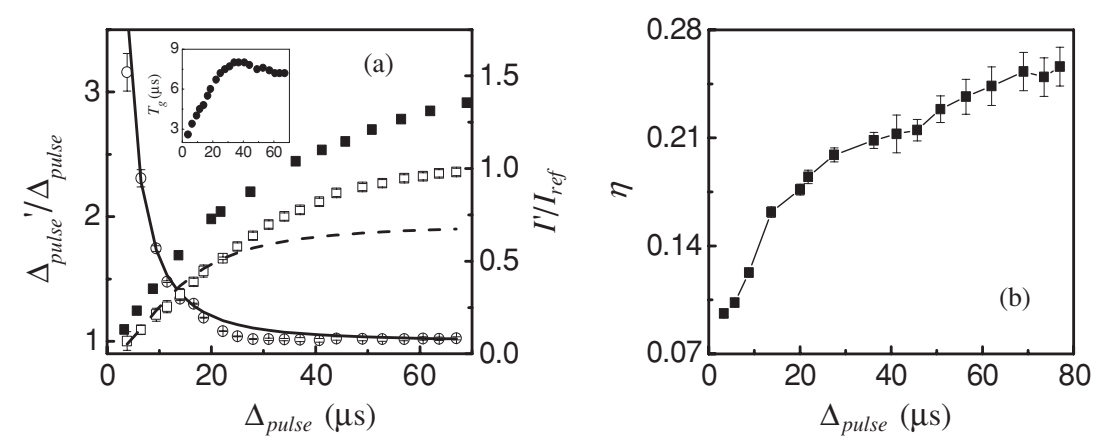

Figure 2. (a) Slowing and signal pulse duration. The broadening ratio data corresponding to the axis labelled $\Delta_{\text {pulse }}^{\prime} / \Delta_{\text {pulse }}$ are marked with (o). The intensity ratio corresponding to $I^{\prime} / I_{\text {ref }}$ is indicated with ( $\square$ and $\square$ ) at different initial signal pulse durations for a cell temperature of about $70{ }^{\circ} \mathrm{C}$. The data marked $\square$ are taken at $5 \mathrm{mG}$ field, while the data marked $\square$ are taken at $<2 \mathrm{mG}$ field. Due to the narrow EIT transparency window, the slowing signal pulse becomes broad and small. The solid and dashed lines are fits to the Fourier transform of the product of a Gaussian pulse with the EIT spectrum. The measured intensity ( $\square$ and $\square$ ) exceeds the prediction because of optical rotation. Inset: pulse delay at different pulse durations. (b) Light storage and signal pulse duration. The storage efficiency is shown and solid line is a guide to the eye.

into real and imaginary parts: $\chi=\chi^{\prime}+i \chi^{\prime \prime}$. For the real part, the index of refraction, $n$, is given by $n=1+\chi^{\prime} / 2$. For the imaginary part, the absorption coefficient, $\alpha$, is given by $\alpha=\omega \chi^{\prime \prime} / 2 \mathrm{c}$. After the light pulse propagates through the atomic vapour cell of length $L$, the intensity of the pulse is attenuated by a factor of $\mathrm{e}^{(-2 \alpha L)}$, which as a function of frequency gives the usual EIT transmission profile. In Lukin and Fleischhauer's paper [3, 15], the EIT transparency window is given by

$$
\Delta \omega_{\text {trans }}=\left(\Omega^{2} / \gamma\right) /(\kappa k L)^{1 / 2} .
$$

The group velocity is defined as, $v_{g}=\partial \omega / \partial k$. Differentiating equation (2) with respect to $\omega$ and assuming that $\chi^{\prime} \ll \omega \partial \chi^{\prime} / \partial \omega$ gives; $v_{g}=\mathrm{c} /\left(1+\omega / 2 \cdot \partial \chi^{\prime} / \partial \omega\right)$. The approximation is justified by equation (1) near resonance. After the light pulse propagates through the atomic vapour cell of length $L$, its envelope is delayed compared to free space propagation by a time $T_{g}=L / v_{g}-L / c=\frac{L}{c} \frac{\omega}{2} \frac{\partial \chi^{\prime}}{\partial \omega}$.

Optical rotation can be understood by considering the two orthogonal circularly polarized components of a linearly polarized beam. The two components couple the two atomic lower states to the upper state in a $\Lambda$-type atom. Consider the case where the excited state has $m=0$ and two ground states have $m= \pm 1$. In the presence of a magnetic field, $B$, along the light propagation direction, the Zeeman effect gives a frequency shift $\pm \mu_{B} B / \hbar$ to the two ground states, where $\mu_{B}$ is the Bohr magneton. Thus the two circularly polarized fields experience two different indices of refraction and optical rotation occurs. The rotation angle, $\Delta \theta$, can be defined as [31] $\Delta \theta=2 \pi / \lambda\left[n\left(\omega_{+}\right)-n\left(\omega_{-}\right)\right] L / 2$, where $\omega_{ \pm}=\omega_{0} \pm \mu_{B} B / \hbar$ and $\omega_{0}$ is the resonant angular frequency.

\section{Results and discussion}

\subsection{Pulse duration}

We first performed the experiment of slow light propagation and storage of light for different signal pulse durations. The results are shown in figures 2(a) and (b), respectively. We emphasize 
that two experimental situations are considered. In the slowing experiment (where the signal pulse is delayed due to propagation through the $\mathrm{Rb}$ cell) the control field is always present, while in the storage experiment the control field is switched off and on. In the slowing experiment, the reference signal pulse duration $\left(\Delta_{\text {pulse }}\right)$ and the reference pulse maximum intensity $\left(I_{\text {ref }}\right)$ are measured off-resonance, and essentially propagate without interacting with the $\mathrm{Rb}$ atoms. The signal pulse duration, $\Delta_{\text {pulse }}^{\prime}$, and the signal maximum intensity, $I^{\prime}$, are measured on-resonance. Both the off-resonance and on-resonance measurements are done with the same laser beam; there is no separate reference beam. From figure 2(a) (open circles), we find that the signal pulse becomes relatively broader at short pulse durations. In addition, the maximum intensity of the signal pulse decreases as the signal pulse becomes broader. This is attributed to increased absorption (squares). The broadening effect is nearly eliminated for pulses longer than $30 \mu$ s duration, while the light intensity still increases slightly with the pulse duration. The inset of figure 2(a) shows the relation of the signal pulse delay time to the pulse duration. It is found that the delay increases with pulse duration and saturates after the characteristic time of $30 \mu \mathrm{s}$. We also found that this characteristic time increases with higher temperatures.

In the light storage experiment, the time sequence of the control and signal fields is the same as in [12] with a storage time of $130 \mu \mathrm{s}$. Light storage efficiency $(\eta)$ is calculated as the ratio of the maximum light intensity between post- and pre-storage (or output and input) after subtracting the leakage from the control beam. The light storage efficiency increases with pulse durations (figure 2(b)).

The above observations are consistent with the following conceptual ideas. Increasing the time duration of the signal pulse leads to a decreasing spectral pulse width. Starting with a very short pulse would mean that the spectral pulse width exceeds the EIT window and the size of the output pulse is very small. The time duration of the output pulse is limited by the Fourier transform of the EIT window. Conversely, when the time duration of the signal pulse becomes very long, its spectral width is much narrower than the EIT window (equation (3)). Consequently the pulse is fully transmitted without broadening.

In our experiment, the incident laser power on our $\mathrm{Rb}$ cell is $2.5 \mathrm{~mW}$, while $0.25 \mathrm{~mW}$ exits after passing through the cell. The laser beam has a $5 \mathrm{~mm}$ beam diameter. The Rabi frequency $\left(\Omega=\sqrt{I \gamma^{2} / 2 I_{s}}\right)$, associated with these laser beam powers is estimated to be about $12 \mathrm{MHz}$ and $3.8 \mathrm{MHz}$, respectively. Here, $I$ is the laser intensity, and $I_{s}=1.6 \mathrm{~mW} \mathrm{~cm}^{-2}$ is the $\mathrm{Rb}$ saturation intensity. The predicted transparency window (equation (3)) is thus bounded by $300 \mathrm{kHz}$ and $30 \mathrm{kHz}$. The experimental data are fitted to the Fourier transform of the product of a Gaussian pulse, $G(\omega)$, and the EIT transmission profile, $T(\omega)$ (figure 2(a), solid line). The Gaussian $G(\omega)$ is the Fourier transform of a Gaussian pulse in the time domain with a width of $\Delta_{\text {pulse }}$ (figure 2(a)). The EIT transmission profile is described in the theory section, and an example of its typical shape is given in figure 3(aiii). The fit gives a transparency window of $50 \mathrm{kHz}$ width. This is within the expected range. The same calculation (assuming an EIT transmission of about 70\% [12]) also gives the maximum output intensity (figure 2(a), dashed line). Because the spectral distribution of the signal pulse $G(\omega)$ is wider than the width of the transmission profile $T(\omega)$ (i.e. the transparency window), the transmitted intensity decreases for shorter pulses (figure 2(a), squares). We would expect that the intensity would exponentially increase with the same time constant as the pulse width exponentially decreased. However, we find that the intensity still increases after the pulse broadening effect stops. This may be due to optical rotation at small but non-zero magnetic fields ( $<2 \mathrm{mG}$, our detector resolution). This suggestion is supported by our observation of even larger light intensities when we increase the magnetic field $(\sim 5 \mathrm{mG}$, filled squares in figure 2(a)). We also find that the temporal delay increases with the pulse durations until 

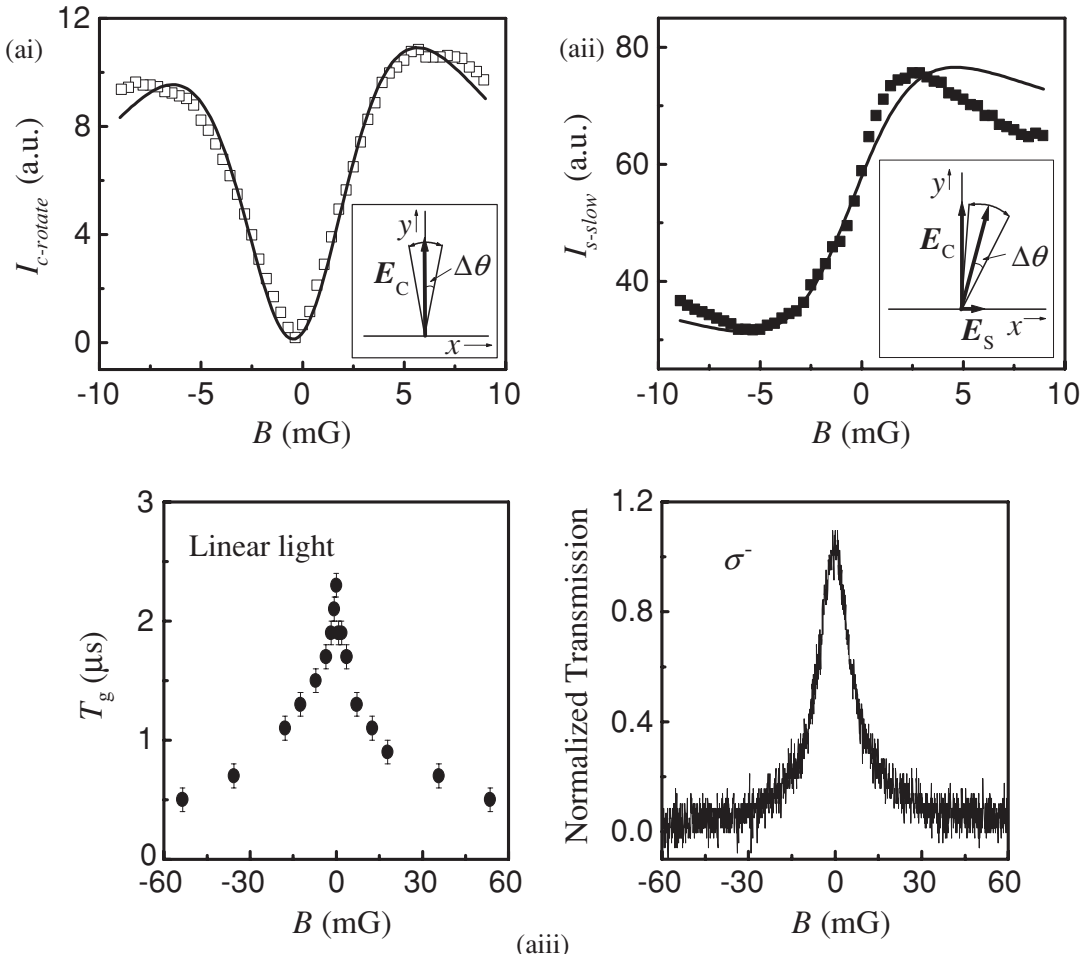

(aiii)
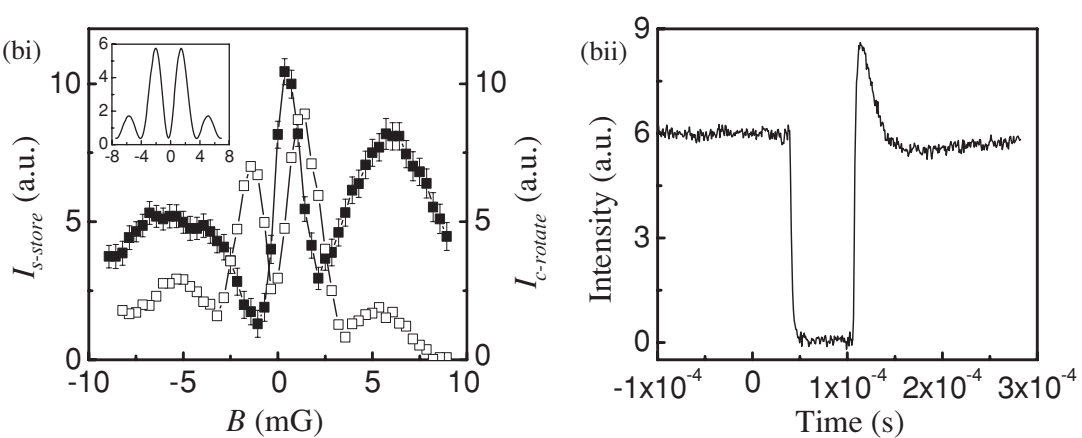

Figure 3. (a) Slowing and magnetic field. (ai) Optical rotation causes intensity in the signal detector from the control light $(\square)$ at steady state. (aii) The peak intensity of the slowing signal pulse ( $\boldsymbol{\square})$ is given as a function of magnetic field. The signal pulse duration is kept at about $15 \mu \mathrm{s}$ and the cell temperature is about $70{ }^{\circ} \mathrm{C}$. Solid lines are the theoretical simulations. Insets illustrate the symmetry when the Pockel's cell is off (no signal pulse), and the asymmetry when the Pockel's cell is on. $\Delta \theta$ is the rotation angle, $x$ denotes the signal light direction, which is parallel to the detector direction, $\boldsymbol{y}$ denotes the control light direction, which is orthogonal to the detector direction. (aiii) The delay of the slowing signal pulse $(\bullet$, left) and the transmission of circularly polarized signal light $\left(\sigma^{-}\right.$, right) are given as a function of magnetic field. The pulse delay is proportional to $\partial \chi^{\prime} / \partial \omega$, while the absorption rate is proportional to $\chi^{\prime \prime}$ (see text). (b) Storage and magnetic field. (bi) The released signal intensity ( $\boldsymbol{0})$ is given after subtraction of the control beam and switch-induced-optical rotation (SIR) ( $\square$ ) as a function of magnetic field for a storage time of $60 \mu \mathrm{s}$. Solid lines are a guide to the eye. Inset: a theoretical simulation of SIR. (bii) A time spectrum of SIR is shown at a magnetic field of $1.5 \mathrm{mG}$. 
the pulse spectral width matches the EIT window (inset of figure 2(a)). For the light storage experiment, a similar effect may cause the observed storage efficiency to increase (figure 2(b)) with pulse duration.

\subsection{Magnetic field}

When a magnetic field is applied along the laser propagation direction, the slow light intensity, $I_{s \text {-slow }}$, shows a dispersion curve as a function of the magnetic field (figure 3(aii), filled squares), while the storage signal, $I_{s \text {-store }}$, shows three peaks (figure 3 (bi) filled squares). The pulse delay is maximum around zero magnetic field (figure 3(aiii), left). When we do not send a signal pulse, but only look at the control light, we see the effect of optical rotation. The intensity in our signal detector due to optical rotation of the control field, $I_{c \text {-rotate }}$, is given when the control light is continuously on and reached steady state (figure 3(ai), open squares). For the steady state the optical rotation signal shows a nice symmetric structure with respect to zero magnetic field. The intensity in our signal detector due to optical rotation of the control field, $I_{c \text {-rotate, }}$, is also given at the time when the control light turns on again (figure 3(bi), open squares). In this case, the optical rotation signal shows four peaks. This means that at non-zero magnetic fields, signals are generated that look like storage signals in the absence of a signal pulse (figure 3(bii)).

Increasing the magnitude of the magnetic field leads to less EIT [12] and more optical rotation (figure 3(ai)). Steady state optical rotation has been extensively studied before (see, e.g., $[7,8])$. Our steady state optical rotation data show a symmetric structure with respect to zero magnetic field (figure 3(ai), open squares). The polarization direction of the detector system is chosen orthogonal to the electric field vector of the control light. Optical rotation of this electric field vector $(\Delta \theta)$ into the direction of the detector explains the symmetry around zero magnetic field (inset of figure 3(ai)). The solid line is obtained from a density matrix calculation for a three level atomic $\Lambda$-system. The calculated optical rotation can be obtained by relating the density matrix coherences to the indices of refraction as experienced by the circular light components of the control light. The small asymmetry in the calculation is obtained by using a slightly different light intensity for $\sigma^{+}$and $\sigma^{-}$.

When orthogonal linearly polarized signal light is present, the optical rotation causes the detected signal to be asymmetric with respect to zero magnetic field as is shown in figure 3(aii) (filled squares). In the case that circularly polarized light is used, a symmetric profile can be observed. The inset of figure 3(aii) illustrates the observed asymmetry for linear polarization. Signal light is generated by optical rotation of the control light in Pockel's cell. Optical rotation due to the $\mathrm{Rb}$ vapour $(\Delta \theta)$ towards the detector direction increases the signal, and vice versa. This feature is also obtained from our model calculation (figure 3(aii), solid line).

We find that the delay of the signal pulse shows a sharp peak at zero magnetic field (figure 3(aiii), left). This peak is similar to the usual EIT absorption profile for circularly polarized light (figure 3(aiii), right). In the theory section the time delay is given by $T_{g}=\frac{L}{c} \frac{\partial \chi^{\prime}}{\partial \omega}$, while the transmitted intensity is given by $\mathrm{e}^{\left(-2 \omega \chi^{\prime \prime} / 2 c L\right)}$. A straightforward functional analysis of the real part $\chi^{\prime}$ and imaginary part $\chi^{\prime \prime}$ of the susceptibility, $\chi$ (equation (1)), shows that both the delay time and the transmitted intensity should exhibit a simple peak structure. In this sense the similar peak structures shown in figure 3(aiii) on the left and right can be understood by realizing that the EIT absorption rate is proportional to $\chi^{\prime \prime}$, while the time delay is proportional to $\partial \chi^{\prime} / \partial \omega$.

Unlike the steady state case, the optical rotation shows some different features when the control light turns on again. A time spectrum shows a peak (figure 3(bii)), which we will refer to as switch-induced-optical rotation (SIR). As a function of magnetic field this SIR gives 

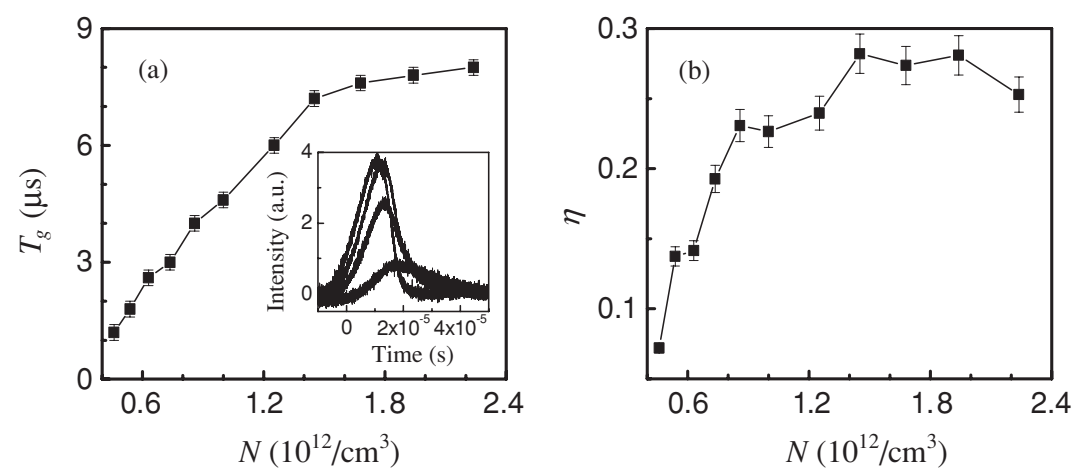

Figure 4. (a) Slowing and temperature. The relation between the density and temperature is given by equation (4). The signal pulse duration is $15 \mu \mathrm{s}$. The solid lines are a guide to the eye. Inset of (a): slowing light propagation at different cell temperatures. The fastest one is off resonance, the others are on resonance, with cell temperature from left to right of $65{ }^{\circ} \mathrm{C}, 75^{\circ} \mathrm{C}$ and $85{ }^{\circ} \mathrm{C}$, respectively. The corresponding atomic densities are $0.5,1.0$ and $2.0 \times 10^{12} \mathrm{~cm}^{-3}$. (b) Storage and temperature. The storage efficiency increases with temperature.

four peaks in our experiment (figure 3(bi), open squares). This result can again be simulated qualitatively from our model (inset of figure 3(bi)). Subtracting the SIR from the regenerated storage signal yields three peaks. These three peaks are due to the storage signal and steady state optical rotation signal. Since SIR affects the storage signal dramatically, one should be very careful when interpreting light storage in the presence of a magnetic field. Further theoretical analysis shows that SIR is sensitive to the experimental conditions, such as atomic density, light intensity, light polarization and spin decoherence time, etc. Even the qualitative features such as the number of peaks is sensitively dependent on these experimental parameters. This situation is different for the case of circular polarization. Then optical rotation does not play a role, which results in clean storage signals.

\subsection{Atomic density}

The effect of different atomic densities on light slowing and storage are shown in figures 4(a) and (b). The total atomic density (with abundances for ${ }^{85} \mathrm{Rb}$ of $72 \%$ and for ${ }^{87} \mathrm{Rb}$ of $28 \%$ ) is estimated from the cell temperature by using Killian's (semi-empirical) formula [32],

$$
N\left(\mathrm{~cm}^{-3}\right)=10^{10.55-4132 / T} /\left(1.38 \times 10^{-16} T\right),
$$

where $N$ and $T$ are the atomic density $\left(\mathrm{cm}^{-3}\right)$ and cell temperature $(\mathrm{K})$, respectively. For the slowing experiment an increased pulse delay is observed for higher atomic densities (figure 4(a)). The inset of figure 4(a) shows the pulse broadening effect for several temperatures. For the light storage experiment the efficiency increases for higher atomic densities (figure 4(b)).

The increased time delay of the slowed signal pulse with atomic density is expected because the expression for $T_{g}$ is proportional to the atomic density. The slowed pulse becomes broader and smaller because, for fixed pulse duration, as the atomic density increases, the EIT transparency window becomes narrower [3, 15]. Conversely, larger pulse delays in the storage experiment lead to larger stored signals (figure 4(b)). For the highest atomic densities the storage efficiency decreases. This is possibly due to enhanced absorption from nearby hyperfine levels. 


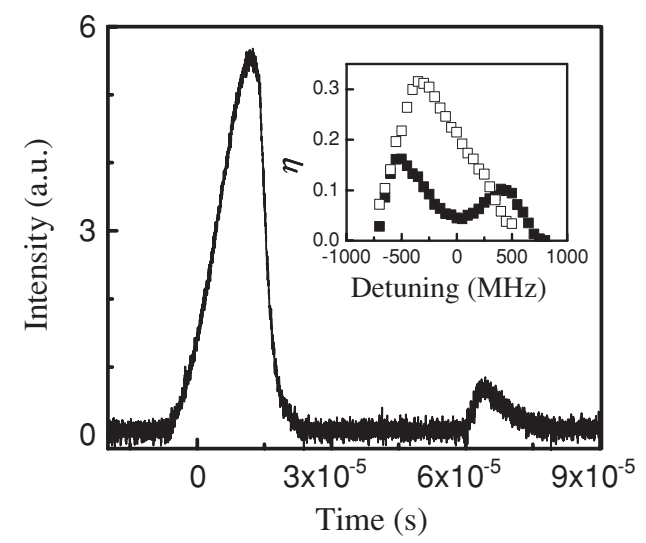

Figure 5. Storage and isotope. Light storage is shown for ${ }^{85} \mathrm{Rb}$ at a cell temperature of $70{ }^{\circ} \mathrm{C}$. Inset: light storage efficiency of ${ }^{87} \mathrm{Rb}(\square)$ at $75{ }^{\circ} \mathrm{C}$ and ${ }^{85} \mathrm{Rb}(\boldsymbol{\square})$ at $70{ }^{\circ} \mathrm{C}$ as a function of laser detuning. Zero detuning corresponds to the transition of $5 \mathrm{~S}_{1 / 2}, F=2 \rightarrow 5 \mathrm{P}_{1 / 2}, F=1$ for ${ }^{87} \mathrm{Rb}$ $(\square)$ and $5 \mathrm{~S}_{1 / 2}, F=3 \rightarrow 5 \mathrm{P}_{1 / 2}, F=2$ for ${ }^{85} \mathrm{Rb}($

\subsection{Isotope}

So far, all the above experiments are done for the ${ }^{87} \mathrm{Rb}$ isotope. Can storage be achieved for the ${ }^{85} \mathrm{Rb}$ isotope? For this purpose we adjust our laser frequency to $\lambda=794.984 \mathrm{~nm}$, i.e., the $5 \mathrm{~S}_{1 / 2}, F=3 \rightarrow 5 \mathrm{P}_{1 / 2}, F=2$ transition for ${ }^{85} \mathrm{Rb}$. The hyperfine structure is similar to that of ${ }^{87} \mathrm{Rb}$. By choosing the operating temperature a little lower than that used for ${ }^{87} \mathrm{Rb}$, we find the light storage signal for ${ }^{85} \mathrm{Rb}$, shown in figure 5. The particular storage time spectrum shown is taken at a laser frequency $-400 \mathrm{MHz}$ detuned from resonance. As a function of detuning, we find that the maximum storage efficiency is usually lower than the storage efficiency for ${ }^{87} \mathrm{Rb}$ (inset of figure 5). Furthermore storage is only observed in a somewhat limited temperature range $\left(60-75{ }^{\circ} \mathrm{C}\right)$. The behaviour of the storage efficiency as a function of detuning is quite different for ${ }^{85} \mathrm{Rb}$ (inset of figure 5). We cannot explain the different behaviour, but we suspect that this is related to the details of the hyperfine structure.

\section{Conclusion}

In summary, we have experimentally studied resonant light pulse propagation and storage in a warm $\mathrm{Rb}$ atomic vapour for different experimental parameters. We find that the light storage signal still exists at very small pulse duration (several $\mu \mathrm{s}$ in our experimental case). However, the slowing signal pulse becomes broad and small. To avoid this effect, the signal spectral bandwidth should be within the EIT transparency window, which we determined to be about $50 \mathrm{kHz}$ in our experiment. The size of the EIT transparency window is found to depend on the vapour temperature. We have measured the magnetic field dependence of the slowing and storage signals. One might expect that requiring the Zeeman shifts to be within the EIT window places sufficiently strong criteria on the strength of the magnetic fields. However, we find that the magnetic fields have to be shielded even better to within $\sim 2 \mathrm{mG}(2.8 \mathrm{KHz})$ to observe a clean light storage signal. The reason is that for linearly polarized light switchinduced-optical rotation (SIR) can mask the storage signal. Storage could be achieved in a temperature range of about $65-90{ }^{\circ} \mathrm{C}$, which corresponds to the atomic density of $0.46 \times$ $10^{12} \mathrm{~cm}^{-3}$ to $3 \times 10^{12} \mathrm{~cm}^{-3}$. For a temperature larger than $90^{\circ} \mathrm{C}$, the storage signal decreases 
due to the absorption. Finally, we demonstrate light storage for the ${ }^{85} \mathrm{Rb}$ isotope within the temperature range of $60-75^{\circ} \mathrm{C}$. It is our hope that the information provided will be of use to the construction of other slowing and storage of light experiments. The knowledge that storage works for ${ }^{85} \mathrm{Rb}$ provides the use of an alternative laser wavelength. The polarization, magnetic field, temperature and pulse duration-dependent studies, provide useful information concerning the selection of optical components, magnetic shielding, temperature control and electronic control.

\section{Acknowledgments}

This work was supported by a Nebraska Research Initiative (NRI) Grant. We thank B Williams for his work on the apparatus.

\section{References}

[1] See, e.g., Harris S E 1997 Phys. Today 5036

[2] Kash M M, Sautenkov V A, Zibrov A S, Hollberg L, Welch G R, Lukin M D, Rostovtsev Y, Fry E S and Scully M O 1999 Phys. Rev. Lett. 825229

[3] Lukin M D, Fleischhauer M, Zibrov A S, Robinson H G, Velichansky V L, Hollberg L and Scully M O 1997 Phys. Rev. Lett. 792959

[4] Zibrov A S, Lukin M D, Hollberg L, Nikonov D E, Scully M O, Robinson H G and Velichansky V L 1996 Phys. Rev. Lett. 763935

[5] Budker D, Yashchuk V and Zolotorev M 1998 Phys. Rev. Lett. 815788

[6] Budker D, Kimball D F, Rochester S M and Yashchuk V V 1999 Phys. Rev. Lett. 831767

[7] Novikova I, Matsko A B and Welch G R 2001 Opt. Lett. 261016

[8] Sautenkov V A, Lukin M D, Bednar C J, Novikova I, Mikhailov E, Fleischhauer M, Velichansky V L, Welch G R and Scully M O 2000 Phys. Rev. A 62023810

[9] Fleischhauer M, Matsko A B and Scully M O 2000 Phys. Rev. A 62013808

[10] Fleischhauer M and Lukin M D 2000 Phys. Rev. Lett. 845094

[11] Kocharovskaya O, Rostovtsev Y and Scully M O 2001 Phys. Rev. Lett. 86628

[12] Phillips D F, Fleischhauer A, Mair A, Walsworth R L and Lukin M D 2001 Phys. Rev. Lett. 86783

[13] Liu C, Dutton Z, Behroozi C H and Hau L H 2001 Nature (London) 409490

[14] Zibrov A S, Matsko A B, Kocharovskaya O, Rostovtsev Y V, Welch G R and Scully M O 2002 Phys. Rev. Lett. 88103601

[15] Fleischhauer M and Lukin M D 2002 Phys. Rev. A 65022314

[16] Mair A, Hager J, Phillips D F, Walsworth R L and Lukin MD 2002 Phys. Rev. A 65 031802(R)

[17] Kozuma M, Akamatsu D, Deng L, Hagley E W and Payne M G 2002 Phys. Rev. A $66031801(\mathrm{R})$

[18] Gao H, Rosenberry M and Batelaan H 2003 Phys. Rev. A 67053807

[19] Bajcsy M, Zibrov A S and Lukin M D 2003 Nature (London) 426638

[20] Patnaik A K, Liang J Q and Hakuta K 2002 Phys. Rev. A 66063808

[21] Bigelow M S, Lepeshkin N N and Boyd R W 2003 Science 301200

[22] Paspalakis E and Knight P L 2002 J. Opt. B: Quantum Semiclass. Opt. 4 S372

[23] Kozuma M, Akamatsu D, Deng L, Hagley E W and Payne M G 2002 Phys. Rev. A 66031801

[24] Imamoglu A 2002 Phys. Rev. Lett. 89163602 James D F V and Kwiat P G 2002 Phys. Rev. Lett. 89183601

[25] Artoni M and Carusotto I 2003 Phys. Rev. A 67011602

[26] Juzeliunas G and Ohberg P 2004 Phys. Rev. Lett. 93033602

[27] Plimak L, Olsen M K and Fleischhauer M 2004 Phys. Rev. Lett. 92253201

[28] Metcalf H J and van der Straten P 1999 Laser Cooling and Trapping (New York: Springer)

[29] Eberly J H, Rahman A and Grobe R 1996 Laser Phys. 669

[30] Payne M G, Deng L, Schmitt C and Anderson S 2002 Phys. Rev. A 66043802

[31] Baak D A V 1996 Am. J. Phys. 64724

[32] Killian T J 1926 Phys. Rev. 27578 